\title{
Review of Elastic: Flexible Thinking in a Time of Change, by Leonard Mlodinow. New York: Pantheon Books
}

\author{
Gurmehr $\mathrm{K}^{* 1}$ and Jonathan $\mathrm{F}^{2}$ \\ ${ }^{1}$ Department of Psychiatry, University of Arizona College of Medicine, USA \\ ${ }^{2}$ Departments of Family Medicine, Larkin Community Hospital, Hialeah, USA
}

*Corresponding Author: Gurmehr Kaur, Department of Psychiatry, University of Arizona College of Medicine, Phoenix, AZ, USA, Email: mehrkaur777@gmail.com

\section{Review Article}

Volume 3 Issue 5

Received Date: June 01, 2018

Published Date: June 13, 2018

\section{Abstract}

In Leonard Mlodinow's 2018 book Elastic: Flexible Thinking in a Time of Change, the author explores what creative thinking is, how to harness it, and aspects of modern society that hinder this kind of thought. Part one of the book explains how people confront change. Mlodinow claims that there is no hard evidence supporting aversion to change, and that in fact all people crave change to varying degrees. The second part of the book examines how we think, categorizing thought into analytical and creative/elastic thinking. In this section, Mlodinow also claims that people are not usually thinking while analyzing new information, but instead running on "autopilot" based on past experiences and expectations. Part three of the book discusses how new ideas are formed. Mlodinow claims that the most creative and paradigm shifting new ideas are formed when the mind is relaxed, at which point it can thinking divergently. He warns against the distractions from technology, as they prevent us from using our idle time to find creative solutions to the problems we face. In the last part of the book, Mlodinow shares strategies on how to think more elastically, suggesting drugs and alcohol as a means to enhance creativity.

Keywords: Elastic Thinking; Analytical Thinking; Compound Remote Associate Problems; Neophiliac; Change Aversion; Bottom up Thinking; Creativity; Technology

\section{Introduction}

Creative thinking is an elusive but vital aspect of societal, artistic, and technological progress. Creativity has been core to the advancement of civilization via revolutions in art, as well as practical problem solving, like creating the first artificial heart valve. Today, there are widespread fears that the technology and its distractions have decreased our ability to think creatively and made us less prepared to handle the new problems that society faces. These are fears are overblown.

In his 2018 book, Elastic: Flexible Thinking in a Time of Change, Leonard Mlodinow makes the argument that "elastic thinking," or thinking creatively for problemsolving, is essential for humanity's ability to thrive in modern times [1]. He states that with the constant 


\section{Psychology \& Psychological Research International Journal}

distractions from technology today, elastic thinking and creativity are made more difficult. He also explores what thinking actually means and how we generate novel ideas. $\mathrm{He}$ ends the book arguing that elastic thinking is necessary to survive in a world of constant stimulation, and puts forth strategies for the reader to make their thought processes more elastic.

Mlodinow espouses that there are two separate forms of thought: analytical and elastic. Analytical thinking is the methodical, step-by-step progression of mental calculations that enables us to analyze information we have collected and make straightforward decisions. Via this mode of thought, we can evaluate and break down complex problems into workable components and manage them in a logical manner. Solving a complex math equation would be an example of this. This form of thinking is referred to in the book as "top down" thinking, and it does not utilize our innate creativity (p. 9).

On the other hand, elastic thinking is a non-linear form of analysis referred to as "bottom up" thinking. It allows us to form ideas that would not stem from a logical, stepby-step process of thought. In this form of thinking, our brains utilize loosely associated concepts, imagination, and recombination of old notions to create new ideas and conclusions (p. 10). Mlodinow gives the example of David Wallerstein who revolutionized the food industry in the 1960s when he suggested larger sizes of popcorn and drinks to improve the profitability of his movie theater. While this seems like an obvious idea nowadays, back then it was an unfamiliar solution to a simple problem. As we can see now, supersizing of portions has become the norm (p. 91).

Mlodinow claims that the rapid rate of innovation and changes in technology has put society under a barrage of distractions. Consequently, we are constantly focused on objects to stave off boredom (p. 124). Social media, video games, books, podcasts, music, and tv shows are always at our fingertips. These limits the idle time the mind is able to wander and evaluate the problems we are confronted with in creative ways. In his book, Mlodinow asserts that although it is perceived that people don't like change, it is in fact a core attribute of the human temperament. He claims there is no such thing as "change aversion" in modern psychology, and thus states that change is not feared, but rather craved by people (p. 19). These claims are only half true. People are usually open to and desire small change; for example, a new car, a new game, a new tv show, etc. However, macro changes, like relocating to a new city, a career shift, or a divorce, are typically feared and cause great anxiety. There is significant data to suggest that aversion to change is central to human anxiety. In fact, the Journal of Anxiety Disorders went as far as to say that, "fear of the unknown may be a, or possibly the, fundamental fear underlying anxiety [2]."

Mlodinow also states that most of the time people run on "autopilot." Their behaviors, social interactions, and thought processes are based on previous assumptions and paradigms without consideration of the new information or the current situation (p. 35). Mlodinow cites a study from the Journal of the American Medical Association (JAMA) showing that experienced doctors may have worse patient outcomes in atypical cases than their novice counterparts (p. 160). However, Mlodinow fails to consider other confounding variables which may cause younger physician to have better outcomes. They are closer to their training so may be more up to date with the latest recommendations, whereas older physicians may not be as aware of new protocols and research. In fact, the British Medical Journal (BMJ) published a study that showing that younger physicians have better patient outcomes in general [3].

In his book, Mlodinow claims that through elastic thinking, new ideas form organically from our unconscious minds. He gives the example of Mary Shelly who was only able to think of Frankenstein when relaxing and letting her mind wander, but when she later actively tried to come up with a plot for a new book, she was unable to (p. 109). Mlodinow refers to the brain's "default network" which allows us to "connect divergent information to form new association... to change the way we frame [problems]... or to generate new ideas (p. 125)." He blames addiction to technology for decreasing creativity in society by limiting idle time in which elastic thinking may occur (p. 124). While it is true that addiction to technology may be a hindrance to creativity, as any addiction would be, it is essential to not forget the ways technology benefits creativity. Studies have demonstrated that creative brainstorming yields better results via an electronic medium than verbal brainstorming by overcoming the space and time constraints of working in a group [4]. Similarly, in one review, social media was shown to increase creativity in higher education [5]. Social media and other brainstorming platforms allow both more time to evaluate a problem and constant communication over a longer period, so unfolding thoughts can take form collaboratively. Cutting edge research has begun to show that creativity in education can even be amplified through the use of virtual reality $[6,7]$. 


\section{Psychology \& Psychological Research International Journal}

After making his arguments on the necessity of elastic thinking, Mlodinow leaves the reader with strategies to help make their minds more elastic. Mlodinow quotes experiments by Kounios and Beeman which observe individuals working through compound remote associate problems (CRA's) while under fMRI or EEG observation. CRA problems are complex word puzzles which cannot be solved through an analytical approach, but instead require elastic thinking. These experiments isolated the anterior cingulate cortex (ACC) as the part of the brain which allows your thinking to broaden and allow more odd and tangential approaches (p. 145). Mlodinow cites another CRA study demonstrating that marijuana improved scores and broadened the ideas of individuals who previously performed poorly. He goes further to claim that alcohol and other drugs increase elastic thinking and creativity and suggests these as strategies for the reader (p. 203, 204). Nietzsche, when commenting on the use of alcohol, wrote "but this people has deliberately made itself stupid, for nearly a millennium: nowhere have the two great European narcotics, alcohol and Christianity, been abused more dissolutely [8]." In reference to alcohol, Nietzsche is saying that it is a intoxicant that numbs the minds, used by people to ignore their problems and stop looking for solutions. This in turn inhibits their creative drives since the problems they face with are able to be brushed aside. While some marijuana use may be shown to increase elastic thinking in controlled experiments evaluating ability to solve word puzzles, this is a far cry from elastic thinking that can be applied to the problems of today.

Change is an ever-present part of the human experience. While the rapid advancements in technology seem to be reshaping how we experience the world every few years, this is simply a slight increase to the rate of change in previous human experience. As Mlodinow suggests, creativity and elastic thinking are indeed an essential part of problem-solving. However, his claim that technology has become a hindrance to creative thinking is incorrect. Technology has changed the world around us for the better. Instead of hindering creativity, it is a tool for amplifying it. A tool of far greater practical use than marijuana or alcohol.

\section{References}

1. Mlodinow L (2018) Elastic: Flexible thinking in a time of change. New York: Pantheon Books.

2. Carleton RN (2016) Fear of the unknown: One fear to rule them all? J Anxiety Disord 41: 5-21.

3. Tsugawa Y, Newhouse JP, Zaslavsky AM, Blumenthal DM, Jena AB (2017) Physician age and outcomes in elderly patients in hospital in the US: Observational study. BMJ 357: j1797.

4. Siau KL (1995) Group Creativity and Technology. The Journal of Creative Behavior 29(3): 201-216.

5. Jahnke I (2011) How to Foster Creativity in Technology Enhanced Learning? Social Media Tools and Platforms in Learning Environments 95-116.

6. Wang X, Lu K, Runco MA, Hao N (2018) Break the "wall" and become creative: Enacting embodied metaphors in virtual reality. Conscious Cogn 62: 102109.

7. Thorsteinsson G, Page T (2007) Creativity In Technology Education Facilitated Through Virtual Reality Learning Environments: A Case Study. Imanagers Journal of Educational Technology 3(4): 74-87.

8. Nietzsche FW (1889) The twilight of the idols. London: Allen \& Unwin. 